\title{
$617-88$ observación microscópica del clínker de cemento portland

\section{Introducción}

Se presenta en esta Memoria un resumen de los trabajos prácticos y experiencias sobre la técnica de observación mineralógica del clínker de cemento portland por medio del microscopio óptico, ejecutados en la División de Química, Sector de Materiales Hidráulícos, del L. N. E. C.

Se describen la preparación de muestras, procesos de ataque y aparatos empleados, y, después de algunas consideraciones generales sobre los clínkeres y sus componentes mineralógicos, se hacen los comentarios pertinentes a las observaciones efectuadas y a las fotografías presentadas.

Se pretende, como resultado de la observación microscópica, averiguar no solamente la constitución del clínker, sino también determinados detalles del proceso seguido en su obtención, tales como la homogeneidad de los crudos, el tiempo de permanencia en la zona de cocción, el grado de combinación de los componentes y los efectos del temple por enfriamiento. Las alteraciones de los componentes cristalinos y la presencia de la cal libre secundaria son, de hecho, bastante significativos.

En todo caso, el examen microscópico se evidencia como un medio de investigación industrial y de análisis, de gran interés para el control de calidad del clínker, ya que pone de manifiesto importantes propiedades que no pueden detectarse por el análisis químico ni por cualquier otro método de laboratorio más sencillo.

Por último, se agradecen las facilidades dadas para la ejecución de este trabajo por parte de los ingenieros don Manuel Rocha, doña Antera Valeriana de Seabra y doña María Onđina Dionisio.

\section{El clínker de cemento}

Esencialmente, el clínker de cemento portland es el resultado de una serie de reacciones en fase sólida o en fase líquida entre óxidos básicos y ácidos a lo largo de un horno. EI tipo de los componentes obtenidos y sus proporciones relativas dependen de diversos y variados factores, desde la composición y la finura de la materia prima y su grado de homogeneización, hasta la forma como se efectúa el enfriamiento, pasando por la temperatura en la zona de cocción y por el tiempo de permanencia en esta zona.

Los componentes principales son los óxidos: silice $\left(\mathrm{SiO}_{2}\right)$, alúmina $\left(\mathrm{Al}_{2} \mathrm{O}_{3}\right)$, óxido férrico $\left(\mathrm{Fe}_{2} \mathrm{O}_{3}\right)$ -ácidos-y cal ( $\mathrm{CaO})$-básico-. La presencia de los óxidos metálicos de aluminio y férrico se justifica, principalmente, por la posibilidad de obtener mexclas eutécticas con punto de fusión relativamen- 
te bajo. Los componentes menores, la presencia de los cuales puede ser perjudicial, son: magnesia (MgO), óxido potásico $\left(\mathrm{K}_{2} \mathrm{O}\right)$, óxido sódico $\left(\mathrm{Na}_{3} \mathrm{O}\right)$ y azufre, bajo la forma de sulfuros. Señalemos, además, que en los cementos portland blancos es esencial, en la medida de lo posible, la ausencia del óxido férrico, responsable de los compuestos coloreacios que, en este caso, son perjudiciales. Esto llevó a estudiąr detalladamente el sistema ternario cal-alúmina-sílice y las composiciones de calcita, caolín y arena, por ser las materias primas camercialmente más puras de los referidos óxidos.

Bien conocidas son las conclusiones prácticas a que se llegó por medio de los liamados módulos silícico, de fundentes e hidráulico, ya que con ellos se dosifican, generalmente, las materias primas de que dispone el fabricante.

El estudio de los diagramas de equilibrio y de las propiedades hidiáulicas de Ios productos obtenidos a partir de diversas composiciones restringe considerablemente el campo del cemento portland. Según estos diagramas se obtienen los siguientes compuestos en equilibrio:

$\begin{array}{cc}\mathrm{SlO}_{2} 3 \mathrm{CaO} & \mathrm{SC}_{3} \\ \mathrm{SiO}_{2} 2 \mathrm{CaO} & \mathrm{SC}_{3} \\ \mathrm{Al}_{3} \mathrm{O}_{3} \mathrm{CaO} & \mathrm{AC}_{3} \\ 3 \mathrm{Al}_{3} \mathrm{O}_{3} \mathrm{LCaO} & \mathrm{A}_{3} \mathrm{C}_{3} \\ \mathrm{AlO}_{\mathrm{O}} \mathrm{Fe}_{3} \mathrm{O}_{1} 4 \mathrm{CaO} & \mathrm{AFC}_{4}\end{array}$

\section{2,1. Composición mineralógica.}

Fueron el francés Le Chatelier (1887) y el sueco Tornebohm (1897) quienes efectuaron los primeros estudios mineralógicos del clínker. Exceptuando algunos detalles de composición, sus determinaciones fueron coincidentes y constituyeron la base de los conocimientos desarrollados hasta hoy dia. Se definieron, según es sabido, cuatro constituyentes principales:

2,1,1. Alita: Formada principalmente por $\mathrm{SC}_{3}$, apareciendo bajo la forma de cristales biaxiales, incoloros, de baja refringencia, con contornos poligonales.

2,1,2. Belita: Preponderantemente formada por $\mathbf{S C}$ se presenta bajo el aspecto de granos redondeados sin forma cristalina bien definida, color castaño y elevada birrefringencia, presentando colores de interferencia. Se presentan estrías con frecuencia que, según determinaciones de Le Chatelier, forman un ángulo de $60^{\circ}$.

2,1,3. Celita: Se cree que consiste en una solución sólida de $\mathbf{A F C}_{i}$ y $\mathbf{A}_{\mathbf{H}} \mathbf{F C}$. Llena los intervalos existentes entre los cristales de alita y belita. Color anaranjado o castaño verdoso. Elevada birrefringencia.

2,1.4. Felita: Se describe como biaxil, incolora $y$ de fuerte birrefringencia, con forma redondeada, estriada o alargada. Posteriormente se ha comprobado que coincide con la belita.

Definió, además, Tornebohm, una masa vitrea isótropa que rellenaba los espacios intercristalinos.

Es necesario advertir que estas determinaciones se realizaron por la observación de láminas delgadas, por transparencia, en el microscopio petrográflco. Posteriormente, el italiano Tavascl (1934) comenzó a emplear la técnica metalográfica, observando muestras pulidas en una sola cara, en un microscopio de reflexión.

\subsection{Preparaelón de las muestras}

En los trabajos de control de laboratorio aparecen dos materiales macróscópicamente diferentes: el clinker y el cemento. El primero se nos presenta, generalmente, bajo la forma de gránuIos, de mayor o menor diámetro, porosos y de elevada fragilidad. El cemento consiste en un material pulverulento con fragmentos de dimensiones improplas para conseguir un pulimento mecánico. Como sea que unas veces nos puede convenir observar un gránulo de clínker completo (por 
ejemplo, para observar la penetrabilidad del azufre procedente del combustible sobre un nódulo) y otras nos convenga estudiar una muestra molida, no siendo coincidentes las técnicas a seguir en cada caso, entendemos preferible tratarlos separadamente.

\section{3,1. Clínker.}

La porosidad y fragilidad a que ya hícimos referencia hacen del clínker un material difícil de pulir convenientemente. Esta dificultad condujo a la técnica de impregnar el gránulo en una resina sintética, transparente, fácilmente polimerizable, de dureza análoga a la del clínker y que, además, no fuese atacada por los reactivos utilizados para el estudio de la muestra.

La técnica es relativamente fácil, pero no es innecesario anotar que exige una atención y cuidados sin los cuales no se obtienen resultados aceptables.

Debe quedar bien sentado el principio de que sin un buen pulimento no hay observación posible.

En un tubo de vidrio de fondo plano se pusieron uno o más granos de clínker (fig. 1); se coloca el tubo en un desecador de vidrio en el cual se efectúa el vacío por medio de la trompa de agua o por una bomba; pasados unos minutos se vierte por la parte superior, con la ayuda de un embudo de decantación, la resina líquida utilizada para la impregnación, en la cual se deberá disolver previamente y en la cantidad necesaria el catalizador apropiado para la polimerización de la resina. Hay que tener especial cuidado en evitar que se forme espuma, debida a la baja presión interna, por lo que se aconseja verter la resina en gotas muy espaciadas, con lo que se previene que el líquido rebose del tubo sin que la muestra quede bien impregnada.

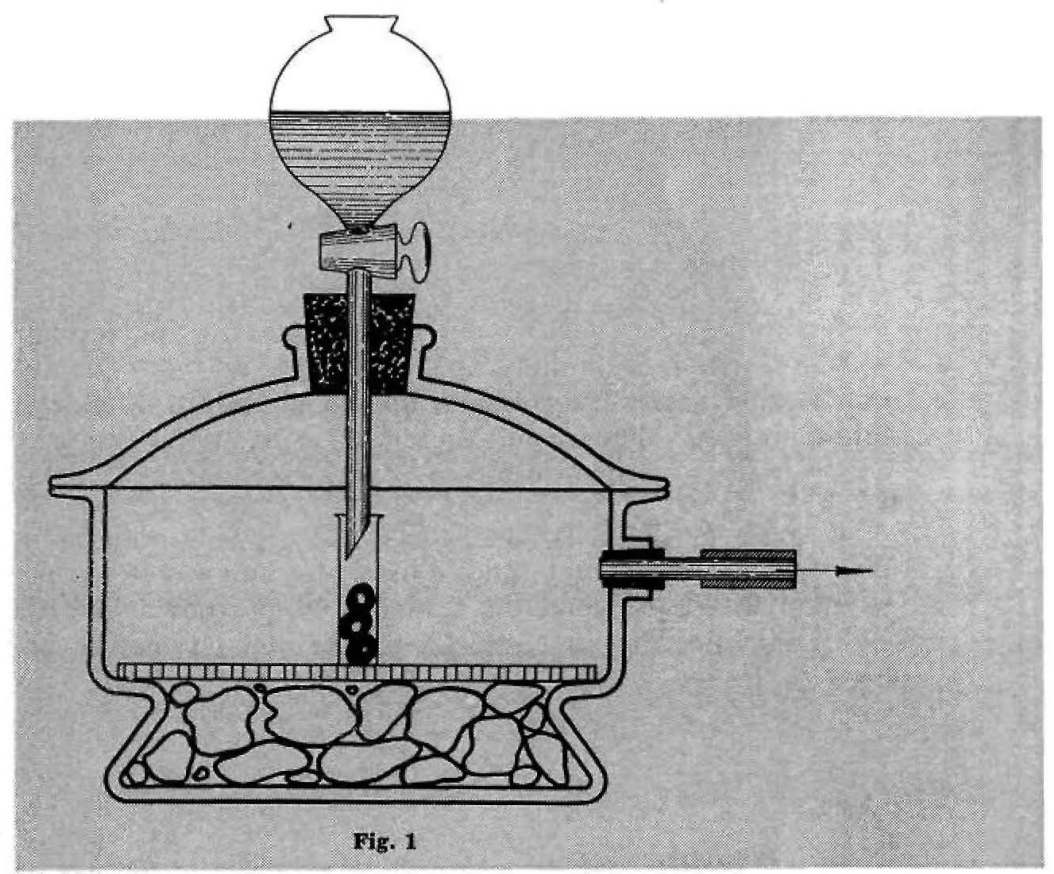

Después de mantener el desecador bajo el vacío durante 15-30 minutos, se retira el tubo y se lleva a una estufa donde permanecerá el tiempo necesario para que la resina polimerice a la temperatura conveniente. Se emplearon dos tipos de resina.

Estireno, con 2 por 100 de peróxido de benzoilo como catalizador. Tarda en polimerizar 24 horas a la temperatura de $60^{\circ} \mathrm{C}$.

Stratil A-16, de Saint-Gobain, con 2 por 100 de Stratil $X-8$ como catalizador. Tarda 4 horas, a la temperatura de $60^{\circ} \mathrm{C}$. Evidentemente, esta resina es más interesante, ya que permite una observación rápida del material, lo que puede tener gran importancia cuando, por ejemplo, se pretende verificar la influencia de una determinada variable en la cocción del clínker.

Una vez polimerizada la resina se parte el tubo de vidrio y se obtiene una pastilla en cuyo interior está el grano, o granos, de clínker. Serrada de forma que se obtenga una sección diametral del grano, se efectúa un primer desbaste, a fin de obtener una superficie plana, en una muela de esmeril, preferentemente horizontal y de velocidad regulable.

El primer pulimento será ejecutado con papeles de lija de números sucesivamente decrecientes, teniendo cuidado de pulir en un solo sentido y en la misma dirección en cada papel. Sólo se debe pasar al papel siguiente cuando se hayan eliminado los restos del papel anterior, siendo 
un procedimiento práctico comprobarlo por observación en un microscopio de reflexión de poca ampliación, o con una buena lupa. Es importante evitar la contaminación de los papeles de lija por las particulas desprendidas de la muestra, por lo que se aconseja cambiar el papel cuando se noten rayas profundas en el pulimento. En nuestros ensayos empleamos papel de lija de esmeril con las finuras $1,0 / 0,3 / 0$ y $4 / 0$.

El segundo pulimento se logra con un fieltro especial sobre el cual se vierte una suspensión de óxido crómico en alcohol absoluto. Moviendo la muestra siguiendo trayectorias circulares se consigue eliminar las rayas de la última lija. Se recomienda tener bastante óxido de cromo en el fleltro y dejar que seque el alcohol hasta quedar levemente húmedo.

Después de estas operaciones la muestra se considera ya pulida, pero con frecuencia se presenta recubierta de gránulos de óxldo de cromo que pueden perjudicar la observación microscópica. Su eliminación es, a veces, difícil y, raramente, completa, pero se aconseja pasar la muestra por un fieltro limpio, seco o humedecido con alcohol absoluto. Se puede prolongar este tratamiento seco durante unos minutos para dejar la superficie más perfecta.

El éxito de todas las operaciones acabadas de describir depende de la práctica del operador, mas difícilmente se podrá pulir la muestra en menos de 30 minutos. Es muy importante usar en estas operaciones alcohol absoluto y, aun así, nunca en cantidades excesivas, pues bastan pequeñísimas cantidades de agua para atacar la cal libre presente en la muestra.

\subsection{Cemento.}

La preparación de una muestra de material pulverulento diflere de la del clínker, ya que, en vez de tener que eliminar una porosidad, se tiene que aglomerar un materlal. Así bastará hacer una mezcla de resina y catalizador en un tubito de vidrio y juntarlo con el polvo sin que ocupe totalmente el volumen del líquido. Es conveniente agitar la suspenstón para homogeneizarla $\mathrm{y}$ expuisar las burbujas de aire que volverian porosa la muestra.

Después de polimerizada la pastilla se puede preparar de dos formas: puliendo la base correspondiente al fondo del tubo, donde lógicamente se encuentran las partículas de mayor tamaño, o serrándola paraielamente a su eje, lo que nos da un campo generaimente mayor y más extenso en tamaños de partícula. La técnića del pulimento es completamente análoga a la descrita para el elínker.

\section{Ataque}

El ataque se realiza, generalmente, por inmersión de la muestra pulida en el reactivo escogido, agitando continuamente, durante el tlempo indicado para cada caso. Terminado el ataque, la muestra se debe iavar inmediatamente en alcohol absoluto si el reactivo estaba en solución alcohólica o en agua destilada si estaba en solución acuosa, pasando después al alcohol absoluto y dejarla secar luego en una corriente de alre. El uso de un paño destruíría los efectos del ataque.

Los reactivos a emplear para el ataque deben ser, en lo posible, específicos de cada componente. Así, cada uno de los constituyentes debe ser atacado, por lo menos, por un reactivo, pues no seria válido identificar uno de ellos por el simple hecho de no haber sido atacado por los reactivos empleados. La elección debe recaer en los reactivos que evidencian la estructura general, sea por el contorno, sea por la diferente intensidad del ataque. Para el caso del clínker y del cemento los reactivos de esta característica serán de carácter ácido, que darán lugar a la formación de sales solubles, pudiéndose usar, indiferentemente, los acidos nítrico y clorhidrico, siempre en solución alcohólica para moderar la velocidad del ataque. Otros reactivos serán específicos, sfendo escogidos admítiendo una inestabilidad diferente de los reactivos ante el reactivo, por lo que se tratará, siempre, de reactivos débiles. En el cuadro que se acompaña se esquematizan los varios reactivos utilizados y sta acción sobre los principales constituyentes del cemento.

Veamos, con más detalle, el efecto de los reactivos corrientemente empleados. 
CUADRO I.-A taque de los componentes del elínker por varios reactivos.

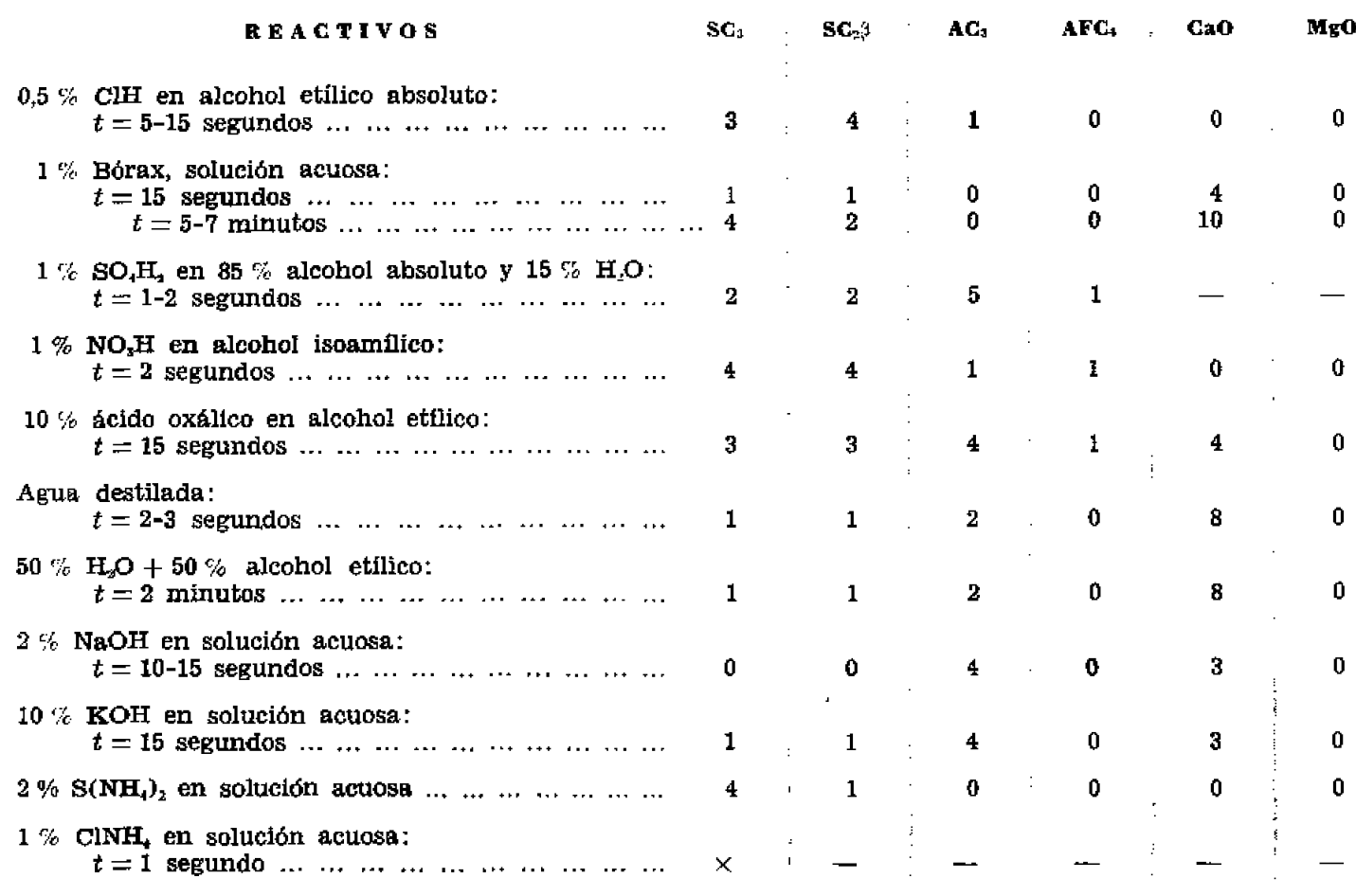

\section{4,1. Solución alcohólica de ClH al 0,5 por 100.}

Ataca a los sillicatos dándoles un color castaño, más oscuro para la belita, permitiendo este hecho distinguirlos entre sí. Evidencia la estrlación de la belita cuando la tiene (fig. 10). Al no atacar la fase intersticial la deja brillante, lo que sirve para delimitar perfectamente los otros constituyentes. La marcación de los límites del gránulo viene facilitada por el hecho de que, siendo esta zona una de las de mayores distorsiones, tiene un alto contenido energético que facilita el ataque del reactivo.

\section{4,2. Solución acuosa de bórax al 1 por 100 .}

Es el reactivo específlco de la alita relativamente a la belita, pero también se acentúa el ataque del solvente (agua) sobre la cal libre, por lo que ésta también se evidencia. Ataca levemente el aluminato de la fase intersticial, dejando brillante el ferrito. Delimita los gránulos. Cuando la cantidad de cal libre en la muestra es considerable, se puede utilizar este reactivo con un tiempo de acción reducido (10-15 segundos) para evidenciarla específcamente.

\section{4,3. Solución de $\mathbf{S O}_{4} \mathbf{H}_{2}$ al 1 por 100 en alcohol de 85 por 100.}

Ataca al aluminato tricálcico dándole coloración castaña, tanto más oscura cuanto más prolongado sea el ataque. Sirve para desdoblar la fase intersticial en aluminato y aluminio-ferrito tetracálcico (figs. $15 \mathrm{y} \mathrm{16}$ ).

\section{4,4. Agua destilada.}

Ataca déblimente al aluminato e intensamente la cal libre. 


\section{4,5. Solución alcohólica de ácjdo oxálico al 10 por 100 .}

Es un reactivo que puede servir de forma diferente según el tiempo de ataque. Así, el primer constltuyente atacado es la belita $y$, luego, Ia alita. Con un tiempo de unos 10 segundos se ponen en evidencia ambos silicatos. Con un tiempo mayor es atacada la alúmina de la fase intersticial ( $t \simeq 20$ segundos) y la fase férrica pierde su brillo, mlentras que los silicatos quedan tan atacados que no permiten la observación de su estructura.

\section{4,6. Ajealis en solución aleohólica (NaOH ó KOH).}

Atacan, preferentemente, el aluminato de la fase intersticial, pero su empleo es raro, ya que se obtienen mejores resultados con el ácido sulfúrico.

\section{4,7. Polisulfuro amónico.}

Ataca y colorea la alita, pero no la belita cuando se encuentra por completo bajo la forma $\beta$, por lo que es un reactivo específco para una de las variedades alotrópicas de la belita.

\section{Observación mieroscópica}

Dividiremos este párrafo en varias secciones para así esquematizar mejor las conclusiones a que se puede llegar de las observaciones. El método que nos parece más conveniente es el de estudiar cada uno de los constituyentes por separado.

\section{5,1. Alita.}

Estudios posteriores a los que llevaron a Le Chatelier a definir este constituyente como si fuese un silicato tricálcico puro, condujeron a una opinión discordante, principalmente por haberse observado anomalías ópticas y por la innegable presencia de una estructura de zonas en algunos cristales de alita. Los diagramas obtenidos por difracción de rayos $X$ conflrmaron la presencia de átomos "extraños", necesarios para explicar la estructura de la alita; son los átomos de Al o $\mathrm{Mg}$, combinados en sus respectivos óxidos. Resuitados recientes dan a la alita la siguiente fórmula molecular:

$$
16 \text { SiO. } \mathrm{MgO} \cdot \mathrm{Al}_{2} \mathrm{O}_{3}, 54 \mathrm{CaO}
$$

correspondiente a una estructura monoclinica con:

$$
a=33,08 \AA ; b=7,07 \AA ; c=18,56 \AA ; B=94^{\circ} 10^{\prime}
$$

Indices de refracción:

$$
\begin{aligned}
& x=1,7158-1,7197 \\
& \gamma=1,7220-1,7238
\end{aligned}
$$

Sin embargo, la actual tendencia de aplicar las teorias de orden-desorden y de resonancia, vuelve cada día menos aceptable la idea de que una estructura molecular fija se vaya repitiendo por todo el cristal.

Como se dijo antes, la alita aparece, generalmente, bajo la forma de cristales bien desarrollados, de contornos hexagonales o prismaticos (flgs. 6, 8 y 10), nítidos cuando no hubo reacciones con la fase liquida durante el enfriamiento. Si esto sucede, se nota una corrosión más a menos intensa, y, dado el carácter básico de los cristales y el carácter anfótero de la fase liquida, debido a la presencia de la alúmina (figs. 4, 6, 10 y 14), el líquido se irá enriqueciendo de sílíce y cal. hasta llegar a una sobresaturación tal que, al proseguir el entriamiento, se originará una cristalización de belita secundaria (figs. 4 y 6 ). 
El campo de estabilidad de la alita esta entre $1.900^{\circ}$ y $1.250^{\circ} \mathbf{C}$; con un enfriamiento brusco a partir de esta temperatura, se obtiene alita en estado metaestable, pero, si el enfriamiento es prolongado, se descompondrá en belita y cal libre:

$$
\mathrm{SiO}_{2} 3 \mathrm{CaO} \rightarrow \mathrm{SIO}_{3} \mathrm{CaO}+\mathrm{CaO}
$$

El aspecto más corriente de esta reacción, en la observación microscópica, es de un grano manteniendo los contornos primitivos, pero moteado con puntos de cal, que se pueden evidenciar con agua destilada, por ejemplo.

Un clínker de composición normal mantenido durante un tiempo bastante proiongado a alta temperatura, o sea, sufriendo un reconocimiento a la temperatura de formación de la alita, aumenta el tamaño de los gránulos de este constituyente a expensas de la fase líquida. En las figuras 14 y 15 se advierten indicios de una cocción proiongada.

Sí una vez formada la alita se enfría el clínker bruscamente, tal como sucede en la fabricación de cemento blanco, los cristales quedarán sujetos a sobretensiones imposibles de compensar por su elasticidad o plasticidad, de donde resultan fracturas irregulares fácilmente observables (figuras 10 y 17).

\section{5,2. Belita.}

Después de la alita, este constituyente es el más importante en el clinker de cemento portland. Cuantitativamente está en menor proporción que la alita, pero, en determinados casos, puede igualar o superar a este constituyente.

Admitia Le Chatelier que estaba formada por $\mathrm{SiO}_{2} .2 \mathrm{CaO}$, pero ya Tornebohm discordaba al darle la composición $\left(\mathrm{SiO}_{2}, 2 \mathrm{CaO}\right)+\left(\mathrm{Al}_{2} \mathrm{O}_{3}, 3 \mathrm{CaO}\right)$, de composición indefinida por consentir soluciones de sustltución; así, cuando las materlas primas son ricas en alcalis, se encuentra una belfta que corresponde a la fórmula $12 \mathrm{SiO}_{2} .23 \mathrm{CaO}, \mathrm{K}_{2} \mathrm{O}$. Cuando consiste en silicato bicálctco puro tiene el punto de fusión a $2.130^{\circ} \mathrm{C}$

Según Ia temperatura a que se mantenga, la belita se puede presentar bajo cuatro formas alotrópicas diferentes, bien deflnidas por Bredig (cuadro II). El paso de una a otra forma tiene lugar a temperaturas diferentes, según sea por calentamiento o por enfriamiento, por los fenómenos bien conocidos de recalentamiento o sobreenfriamiento necesarios para la aparición de la entalpia libre suflciente para vencer la resistencia a la transformación, función de las diferentes entalpías libres de las dos formas y de la resistencia a la difusión a cada temperatura.

CUADRO II.-La fase silicato bieáleico.

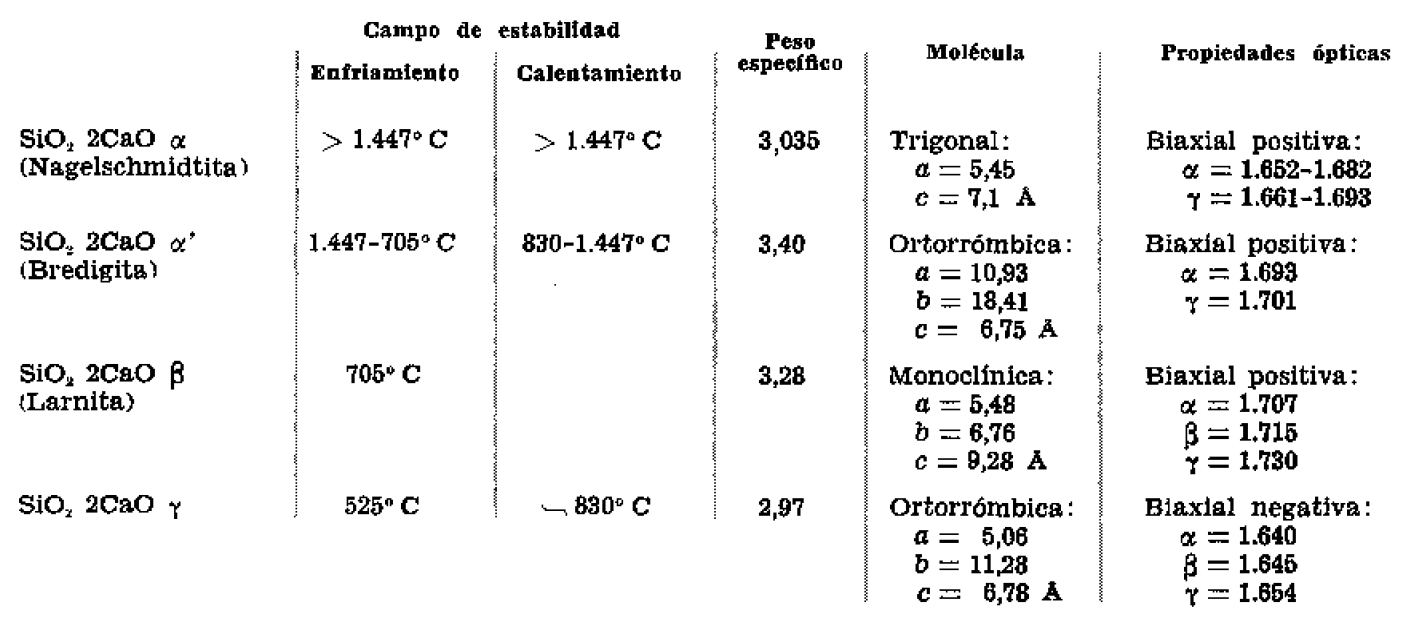

Dada la elevada temperatura de estabilidad de la forma $\alpha$ es fácil comprencier la dificultad en obtenerla; aún con un enfriamiento muy rápldo necesita la presencia de aditivos estabilizadores como $\mathrm{Na}_{2} \mathrm{O}$ y $\mathrm{Al}_{2} \mathrm{O}_{3}$. 
A Ia forma $y$ le fueron inicialmente negadas propiedades hidráulicas. Su presencia es causante de la pulverización espontánea de los cristales de belita debido a la diferencia de pesos específicos entre las formas $\beta$ y $;(0,31)$, de aqui que se evite el enfriamiento lento del clínker.

Por exclusión de partes, concluiremos que, como realmente sucede, las formas $z^{+}$y $\beta$ son las más frecuentes en el cemento portland. La forma $\alpha$ cristaliza en el sistema rómbico y se presenta, en general, en cristales de sección redondeada. En la superfleie es frecuente observar dos series de estrias, de explicación dudosa, pues si admitimos que las líneas más claras corresponden a la forma $\beta$, tendremos que el estríado será consecuencia de la reacción alotrópica; pero también se ha emitido la hipótesis de que las agujas oscuras, principalmente cuando presentan un aspecto punteado (figs. 5 y 11), son el resultado de la segregación de sustancias inicialmente en solución en la forma $\alpha$, luego las líneas blancas $(\beta)$ irán creciendo a costa de las negras, reduciendo éstas a pequeños glóbulos si el tiempo de permanencia a la temperatura de reacción fuera suffelente. De conformidad con el porcentaje de las áreas correspondientes a las estrías blancas y a las estrias negras será posible evaluar el grado de transformación y, por consecuencia, la velocidad de enfriamiento. Una cocción prolongada puede favorecer, también, la aparición de líneas de segregaclón irregulares (flgs. $10 \mathrm{y}$ 12), llegando hasta la forma llamada "dedo de guante" (flgs. 14 y 16), resultado de la completa segregación de las sustancias disueltas, además de una reacción de disolución con la fase Íquida. Bajo este último aspecto es casi inexistente la germinación polisintética, mostrando puntuaciones negras. Sefialamos anteriormente el hecho de la aparición de cristales secundarios de belita resultantes de la disolución de la alita en la fase lfqulda; esta belita cristaliza en pequeñas puntillaciones que crecen Iongitudinalmente con un aspecto levemento prismático, pero muy apartado del característicamente redondeado de la belita $y$, de otra parte, muy semejante al "dedo de guante". Esto nos puede llevar a admitir que esta última forma pueda tener precisamente origen en una recristalización de una alita anterior en una belita secundaria.

Otro hecho interesante observado es la coexistencia en un solo cristal de la alita y la belita (ftgura 13). La explicación del hecho dependerá de cada caso particular; pero si, por ejemplo, tuviéramos un campo de belita en el centro de un cristal de alita, cuyo contorno es, sin embargo, belítico, podemos admitir que inicialmente se formó un cristal de belita durante las reacciones en estado sólido a la entrada de la zona de cacción, siendo dificultado su crecimlento por verse posteriormente envuelto, ya en las reacciones con fase líquida, por un medio más rico en cal y, por lo tanto, propenso a la formación de alita. La presencia de estrías en la inclusión de belita nos indica que, iniclalmente, estaba en la forma $\alpha$, dándose más tarde la inversión para la forma $\alpha^{\prime}$, que-como sabemos-admite menos soluclones sólidas en su seno.

\section{5,3. Fase intersticial.}

El ligante entre los diferentes cristales de alita y belita fue inicialmente clasiflcado como un componente mineral bien definido, la cellta; posteriormente, sin embargo, se abandonó este concepto por comprobarse que estaba formado por dos constituyentes principales: el aluminato tricálcleo $\left(\mathrm{AC}_{3}\right)$ y el alúmino-ferrito tetracálcico ( $\mathrm{AFC}$ ).

Es en la fase intersticial donde se quedan en solución sólida la mayor parte de los componentes extraños, y de aquí que su composición no sea la que corresponde al caso de equilibrio. Tiene su origen en la fase líquida presente en la zona de cocción y es fácil de imaginar que, cuanoo la velocidad de enfriamiento permite a sus componentes adquirir una estructura cristalfna, verá sus formas limitadas por los cristales ya formados de slilcatos. Cuando Ia velocidad de enfriamiento es tan rapida que la fase líquida solidifica metaestablemente, no será correcto clasificarla como siendo un "vidrio".

Se considera nulo el poder hidráulico de la fase férrica y el de la fase aluminato apenas es senslble en los primeros dias después de la preparación de la pasta cementicia y tan sólo en el caso del clínker estar templado.

5,3,1. Aluminato. Se le conoce, generalmente, con el nombre de fase intersticial oscura por su menor poder reflector y mayor reactividad con los acidos, razón por la cual toma una coloración más oscura, gris con ClH (flg. 2) y negra con $\mathrm{BO}_{4} \mathrm{H}_{2}$ (flgs, 5, 6, 14, 15 y 16). Cristalizando entre los silicatos ya formados presenta, generaimente, formas irregulares; pero si el campo y la velocidad de enfriamiento lo permiten, cristaliza en prismas rectangulares (flgs. 15 y 16). Esta es una 
característica prácticamente especiffca, que nos permitirá concluir sobre el sistema de enfriamiento existente en el horno donde se obtuvo el clinker observado, recordando que un enfriador de parrilla con ventilación forzada provoca un gradiente de temperatura mayor que un enfriador rotativo, planetario o no, con circulación natural de aire.

Cuando las materias primas son ricas en álcalis y el enfriamiento es sufleientemente lento, el aluminato cristaliza en largos cristales aciculares característicos. Por tratarse de una anomalía se identíficó esta forma como siendo un compuesto diferente $\mathrm{A}_{2} \mathrm{C}_{3} \mathrm{Na} \mathrm{a}_{2}$, pero, posteriormente, se comprobó que se trataba realmente del aluminato tricálcico.

De acuerdo con afirmaciones anteriores, tendremos que la presencia de la fase intersticial bajo la forma vitrea estará acompañada de la existencia de cristales de alita con contornos bien definidos.

5,3,2. Alúmino-ferrito tetracklcico. Es en este constituyente donde se encuentra la totalidad del hierro, tanto si entró comblnado con las materias primas como si fue agregado como fundente (cenizas de pirita, p. e.), pues el hierro sólo puede aparecer libre, en pequeños nódulos en e] interior de los gránulos de clínker, cuando la cocción se ha efectuando en una atmósfera fuertemente reductora. La fase férrica presente en el clinker de cemento portland es, generalmente, el alúmino-ferrito tetracálcico (brownmillerita), término medio de una serie de soluciones sólidas de aluminato en ferrito cálcico, siendo $\mathrm{FC}_{2}$ y $\mathrm{A}_{2} \mathrm{FC}_{0}$ los términos extremos. De conformidad con el módulo de fundentes $(A / F)$ se admiten mayores o menores porcentajes de otros términos sin ser el $A F C$, si bien éste es siempre el que predomina.

Dada la composición de las materias primas, este componente no estará presente en el clínker para cemento portland blanco (fig. 6). Los inevitables vestigios de hierro están imposibilitados de cristalizar bajo la forma de ferrito si se somete el clínker a un temple enérgico a la salida de la zona de cocción, con lo que se mantiene en solución sólida metaestable.

Su estructura es complementaria de las anteriores, así tanto puede aparecernos de forma irregular relienando los intervalos entre los silicatos, como de aspecto cristalino, con tendencia para la forma prismátlea (figs. 15 y 16).

\section{5,4. Componentes accidentales.}

5,4,1. Cal libre. Reconocida como casi inevitable su presencia en el clinker, sea por deflciencias en la molienda o en la homogeneización de las materias primas, sea por cocción defectuosa o por otras causas, se trata en todo caso de óxido cálcico que no se combinó durante su paso por el horno. Es invariablemente indeseable para los cementeros, puesto que, si innegablemente posee propiedades hidráulicas, sufre infelizmente un conocidisimo aumento de volumen al hidratarse que causa no pocas preocupaciones al fabricante. For esta razón es de interés primordial la identificación de su presencia en la rutina laboratorial del control del horno, $y$, si son universalmente atilizados los procedimientos de ia quimica analitica, creemos que no son de despreciar las grandes posibilidades que ofrece la observación con un microscopio de reflexión de pequeñas dimensiones sin platina integradora.

La cal libre puede presentarse bajo dos aspectos diferentes según sea su origen, La cal libre primaria se presenta bajo la forma de granos redondeados (flgs. 7, 9 y 19), con dirametro del orden de unas centésimas de milímetro. Resulta de una eventual sobredosificación en carbonatos del crudo. Entonces, aun después de una cocción prolongada, se encuentran los gránulos diseminados. Cuando la cocción es insuftctente también nos aparecerá cal libre, resultado del COCa que, si błen descarbonató, no legó a reaccionar con la sílice por falta de oportunidad, apareciendo simultáneamente gránulos de sillce (cuarzo) en la muestra. Las deficiencia en la molienda o en ia homogeneización conducen a la aparición de colonias de nódulos de cal libre; estas condiciones pueden ser parcialmente corregidas cuando la composición potencial de la materia prima es la correcta, por medio de una cocción prolongada, que facilitará la difusión de los óxidos.

La cal libre secundaria, conocida también por cal aérea, es el resultado de la descomposición de la alita cuando se da una permanencia prolongada del clínker a una temperatura próxima de Ios $1.250^{\circ} \mathrm{C}$. Aparece como pequeñas inclusiones, del orden de la micra, diseminadas por el cristal de alita y en mayor proporción por los contornos, ya que allí, por haber una mayor concentración de energía libre, fue más acentuada la reacción de descomposición (flg. 4). 
Los reactivos generalmente más indicados para evidenciar la cal libre son la mezcla alcoholagua (fig. 9), el agua destilada y la solución de bórax (fig. 19); pero no queremos dejar de señalar que basta un buen pulimento de la muestra para revelar los gránulos de cal libre primaria, que se presentan muy brillantes (fig. 7).

5,4,2. Magnesia libre. El empleo de calizas dolomiticas como materia prima para la fabricación de clinker de cemento portland, introduce el magnesio como nuevo elemento en la mezcla reactiva. Dadas sus propledades químicas y sus dimensiones atómicas muy semejantes a las del calcio (recordemos que ambos están en el mismo grupo de la clasiflcación períbdlca de los elementos), es fácil admitir que un pequeño tanto por ciento de $\mathrm{MgO}$ en el crudo será dificll de observar microscópicamente, pues da soluciones solidas de sustitución en los silicatos o permanece en el vidrio sí el enfriamiento del clínker es rápido. De la comparación entre los resultados del análisis químico y del recuento microscópico, por medio de la platina integradora, se llegó a la conclusión de que, aproximadamente, 1 por 100 de $\mathrm{MgO}$ permanece en solución sólida.

Cuando cristaliza individualmente lo efectúa bajo la forma de periclasa (MBO), mineral eúbico que presenta pequeños cristales de contorno generalmente cuadrangular $(0,1,1)$ o triangular $(1,1,1$,$) y gran dureza. Precisamente debido a su dureza es por lo que se puede identificar fáciI-$ mente, pues con la muestra pulida y sin ataque es observable por su gran brillo y la diferencia de nivel en relación al plano de la muestra. Estos cristales aparecen slempre en el seno de la fase intersticlal, por ser el último componente a cristalizar, lo que hace que, si se somete el clinker a un temple muy violento, cristalice en pequeños cristales submicroscópicos y, desde luego, inobservables.

5,4,3. Sulfatos. Cuando el tiempo de permanencia del clínker en la zona de cocción es muy prolongado, tal es el caso de los ladrillos refractarios preparados con clínker de cemento, bolas o anillos accidentalmente formados durante el proceso de la cocción, hay una absorción del anhidrlao sulfuroso resultante de la combustión del carbón o de los aceites pesados usados para el calentamiento del horno, reaccionando con la cal libre primaria o secundaria del clínker, obteniéndose, en presencia de oxígeno, cristales prismáticos de sulfato cáicico, que se pueden evidenciar microscóplcamente con un ataque por $\mathrm{ClH}$, resultando blancos debido a su inatacabilidad.

5,4,4. Sulfuros. Los sulfuros son prácticamente inexistentes en los clinkeres normales de los hornos rotativos, pero pueden encontrarse en los clínkeres de hornos verticaies. Sin embargo, es un hecho su presencia anormal, lo que se puede explicar por dos mecanismos: por la existencia en el horno rotativo de una zona comprendida entre las zonas de descarbonatación y de cocción, donde el sulfato cálcico eventualmente presente en el crudo se descompone con liberación de $\mathrm{SO}_{2}$, el cual es arrastrado por los humos, reaccionando luego con la cal procedente de la descarbonataclón de la callza, regenerando el sulfato:

$$
2 \mathrm{SO}_{4} \mathrm{Ca} \rightarrow 2 \mathrm{SO}_{3}+\mathrm{O}_{2}+2 \mathrm{CaO}
$$

Existe asi un proceso cíclico de este compuesto en esta zona $y$, si por una incidencia en la marcha del horno, algún sulfato consiguiera pasar a la zona de cocción sin cescomponerse, pudiendo reaccionar con el combustible para dar lugar a sulfuro:

$$
\mathrm{SO}, \mathrm{Ca}+2 \mathrm{C} \rightarrow 2 \mathrm{CO}+\mathrm{SCa}
$$

\section{Dureza de los constituyentes}

Una de las primeras anotaciones interesantes que se hacen al observar una preparación microscópica obtenida a partir de un cemento, o de un clinker molido, es que la fractura se da más frecuentemente transcristalinamente para la alita e intercristalinamente para la belita, mejor dicho, la alita se quiebra por la mitad del gránulo, pero la belita es menos frágll que la fase intersticial, por lo que se fragmenta por fractura de esta última (figs. 5, 10, 11, 12 y 13).

Como sea que las operaciones de la molienda son las económicamente más dispendiosas en la industria del cemento, creemos que seria de gran interés el estudlo de la dureza de los componentes del clinker mediante un microdurómetro adaptable al microscopio, lo cual, sin embargo, se sale de los límites del presente trabajo. 


\section{Comentarios a las microfotografías}

Fig. 2. Para poder determinar el tiempo de exposición necesario para la conveniente impresson de la placa refleetividad de la muestra, se hizo una exposición con desplazamientos progresivos de la lámina metálica que cubre la placa.

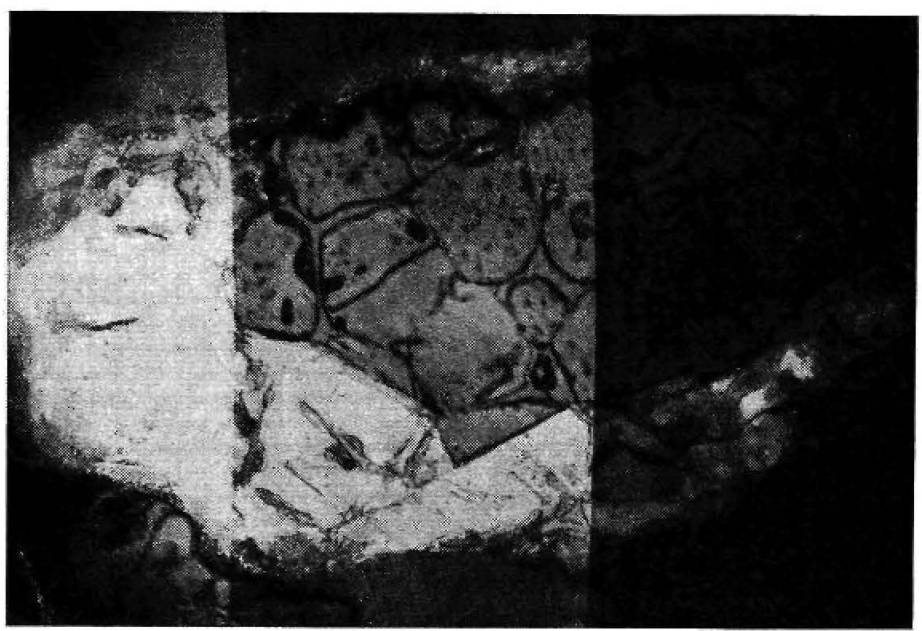

Fig. 3. CLINKER PARA CEMENTO BLANCO.-Grano con cristales de alita de bordes bastante bien definidos, mostrand aigunas corrosiones; fase intersticial abundante $y$ grandes granos de cal ibre. Corresponde a una zona de sobresaturación de cal, pues los compuestos adyacentes a la cal libre son los mas $\mathbf{y} \mathbf{A C}_{3}$ )
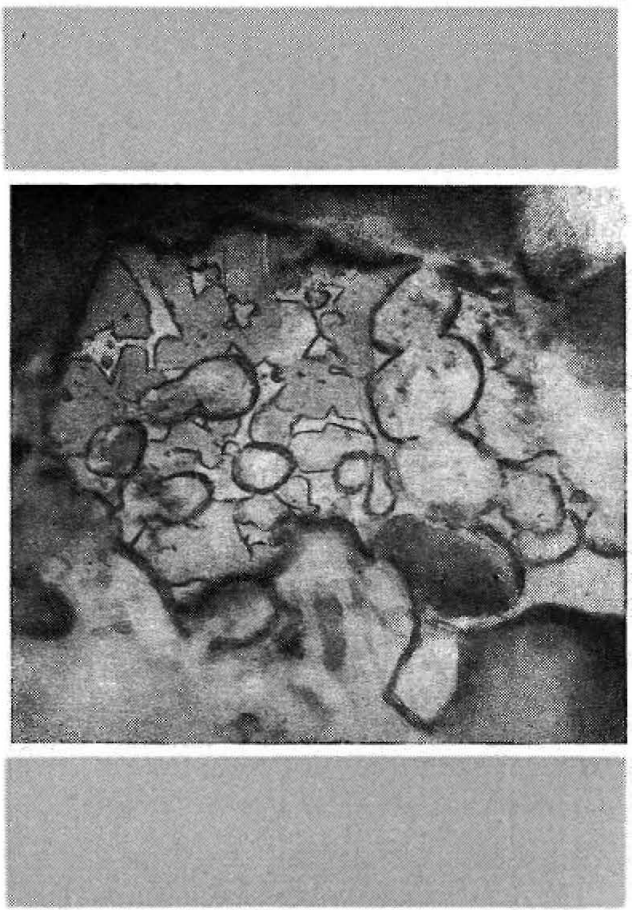

Fig. 4. CLINKER PARA CeMENTO BLANCO.-Zona comcesulares debido a la descomposición de la alita en belita y cal libre secundaria, resultado de una permanencia prolongada a alta temp de coccion. Notanse cristales pequeños de belita recristalizados de la fase liquida. El aluminato separa completamente los cristales de silicatos. El gránulo desenfocado es óxido crómico,

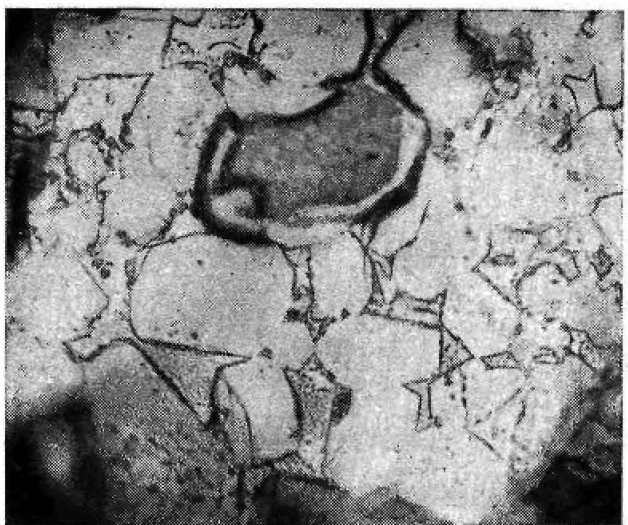



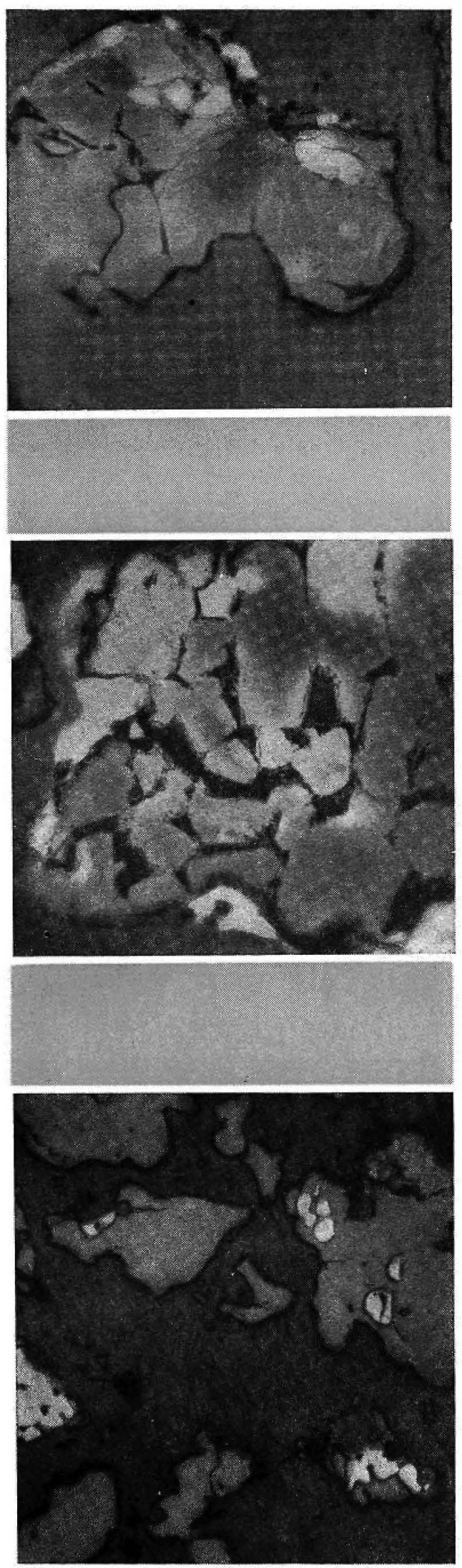

Fig. 5. GLINKER PARA CEMENTO BLANCO-Fragmento de clinker completamente belitico $y$ con poca fase intersticial; la belita continua hacia un campo alitico. Se notan las estrias marcadas por puntuaciones, lo que corresponde a un peque os una cocción lenta para este clínker.

Fig. 6. Clinker Para Cemento blanco.-No es observable la presencia de ferrito en la fase intersticial, como era de esperar. Los silicatos están, generalmente, bien cristaliza dos, notandose la presencia de bastante fase interstieial y la de la descomposición de la alíta. Es notable la disposición de estos eristales de belita, tanto en esta fotografía como en ia número 3 , pues nos llevan a admitir una orientación y una ordenación.

Fig. 7. CLINKER PARA CEMENTO BLANCo.-La observación visual de la muestra, que presenta gránulos de cal libre formando pequeños aglomerados en el seno del clinker, no de eal pensar antes en la existencia de una sobresaturación do 
Fig. 8. CLINKER PARA CEMENTO PORTLAND NORMAL. Nótase una zona predominantemente belítica, Bastante fase interstieial. Cristalización más desarrollada de la alita, pero bastante regular, dentro de los límites normales. Se puede admitir que el clinker sufrí un paso muy rápido de la zona de cocción a la de enfriamiento, dado que no se presentan síntomas eualesquiera de recalent
permanencia a altas temperaturas.

Fig. 9. Clinker PARA CEMENTO BLANCO-Comentario idéntico al de la figura 7 siendo de notar el ataque bien idéntico al de la figura 7 , siend

Fig. 10. GLINKER PARA GEMENTO BLANCO,-La placa está algo desenfocada, pero se notan perfectamente las fraeturas surridas por la alita $y$ principalmente por la belita cenition abun ab́nulo de alita de una aureola probablemente de la en un posieión de la alita a partir de un punto conteniendo una impureza cualquiera, o de un alto contenido energético que le confríó gran inestabilidad, comparable a la de los límites del gránulo.
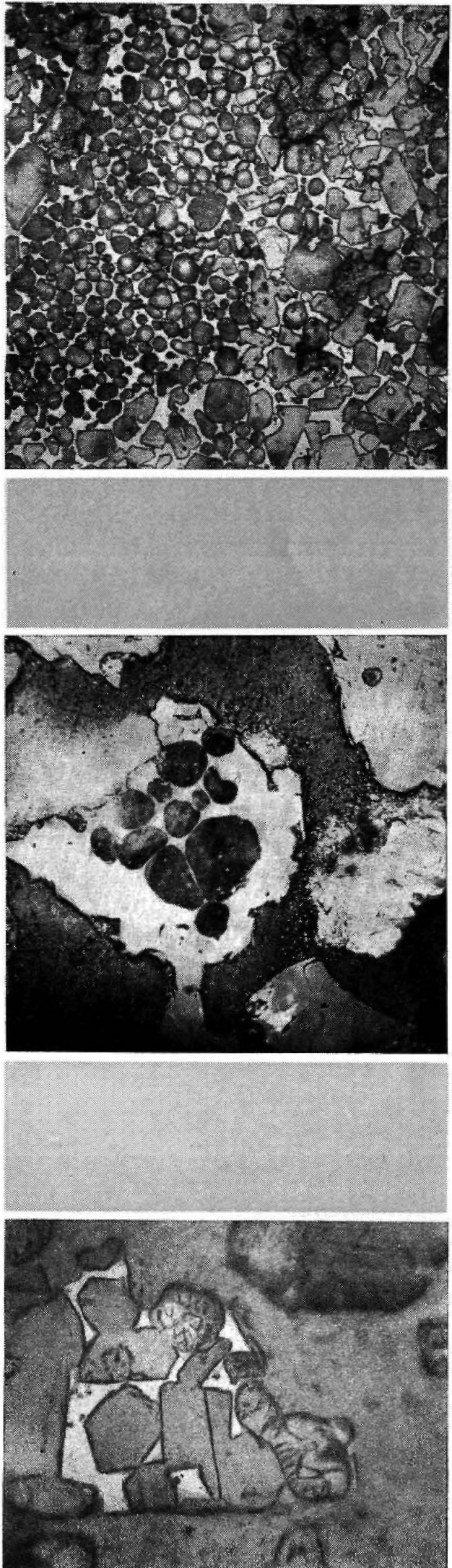

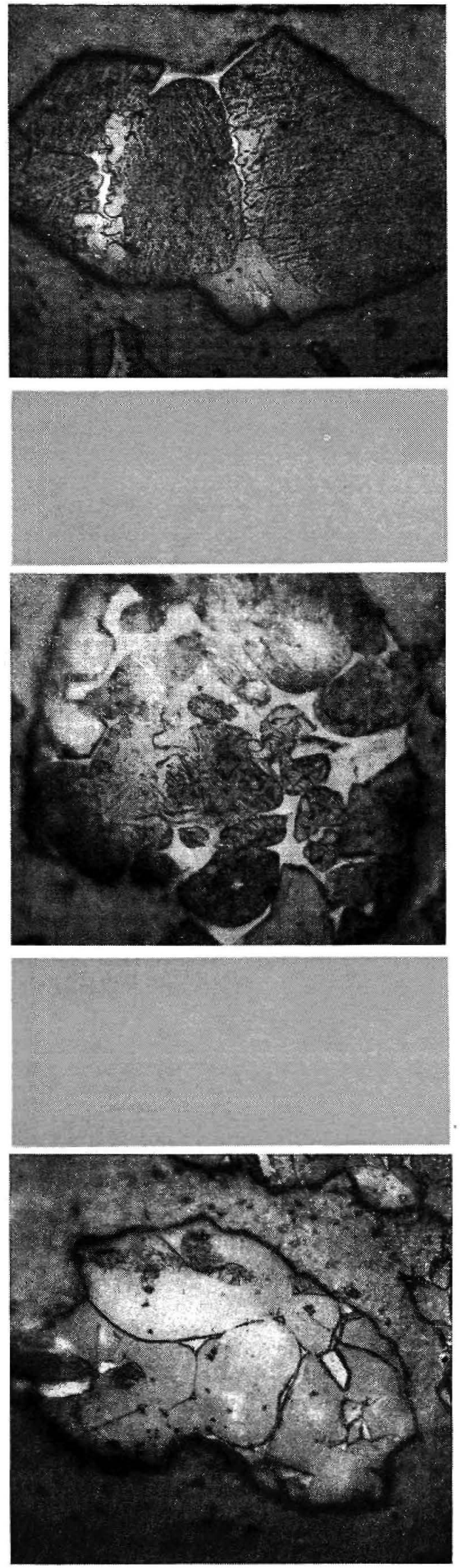

Fig. 11. CLINKER PARA CEMENTO BLANCO.-Fragmento de clínker predominantemente belítico. La belita presents niidas direcenon de inversion, pero con la sustitucion de las es figura 5. Ia fase intersticial es poro abundante, En alsunos punto puntos de perifín intersticial, cuando el crecimiento del grano. En la parte inferior de la fotografía se ven perfectamente dos granos de belita soldados por un grano de alita, de formación anterior.

Fig. 12. GLINKER PARA CEMENTO BLANCO.-Zona belitica, donde aún son visibles las direcciones de germinación. pero que, por una muy avanzada segregación de oxidos, la belita aparece muy hendida. Se ve bastante fase intersticial. La placa está muy desenfoeada. Fig. 13. CLINKER PARA CEMENTO BLANCO.-Belita trans-
formada en alita, conservando aún los contornos redondeados formada primitivos de inversión. nucles de bella, donde se notan las descomposición en belita y cal libre, como es perceptible por la corrosión en los límites del grano. Es de señalar la pequeña cantidad de fase intersticial presente. 
Fig. 14. Clinker PARA Gemento PORTLAND NORMal. Redisolucion de la belita en la fase líquida, tomando en al. gunos puntos el aspecto de udedo de guantes. Fase interstieial gada.

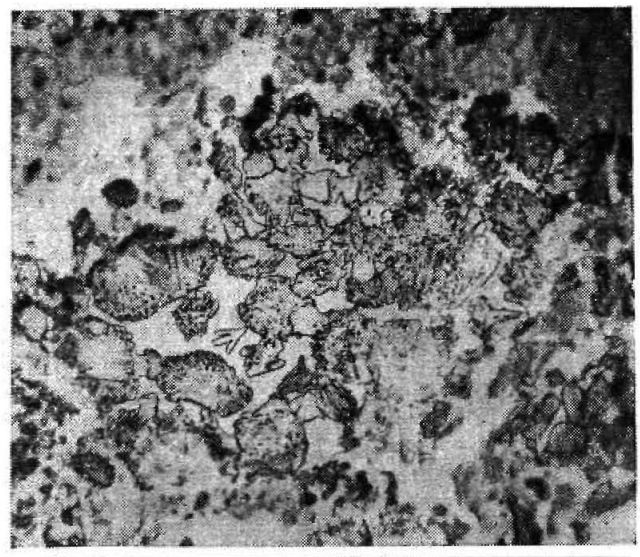

Fig. 15. CLINKER PARA CEMENTO PORTLAND NORMAL. tos (oscuros) en la fase Se notan los silla rancia de la intersticial.

Fig. 16. CLINKER PARA CEMENTO PORTLAND NORMAL. Aluminato con tendencia a cristalizar prismáticamente. Redisolución de algunos granos de belita y recristalización de distibu colita formados, pero mostrande una coceión excesiva. La placa esta
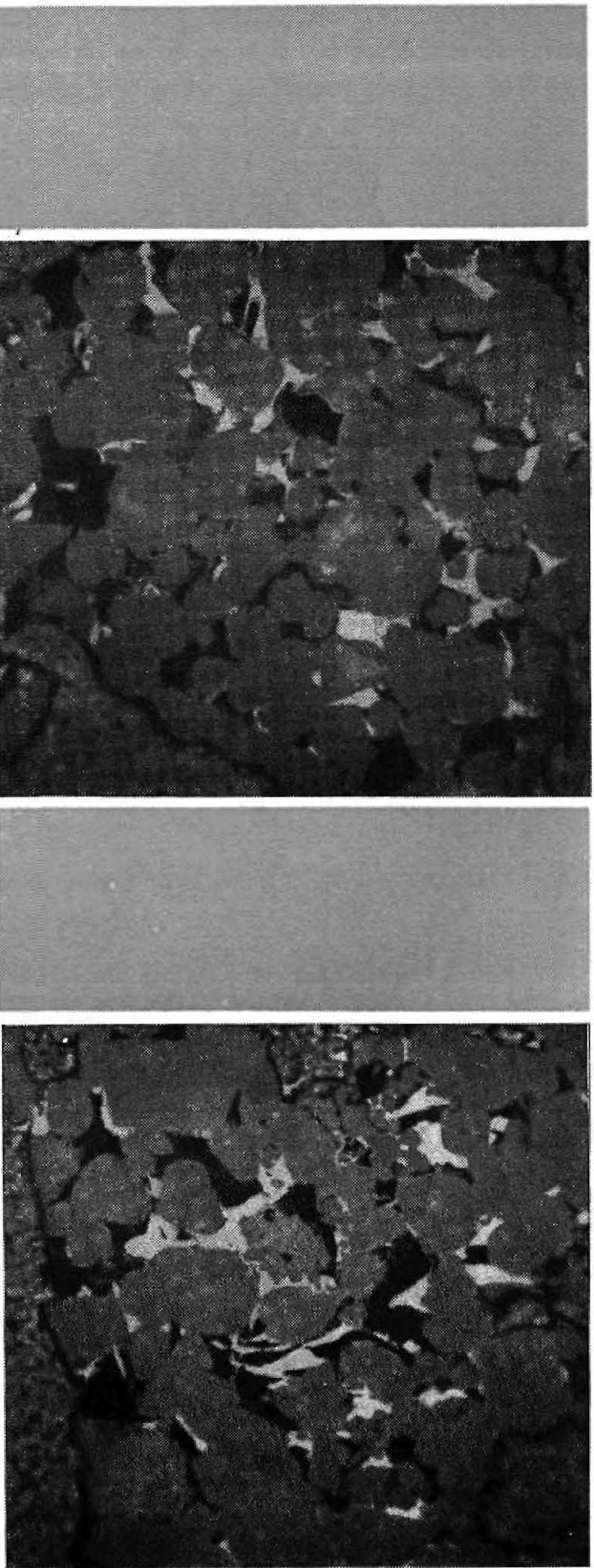

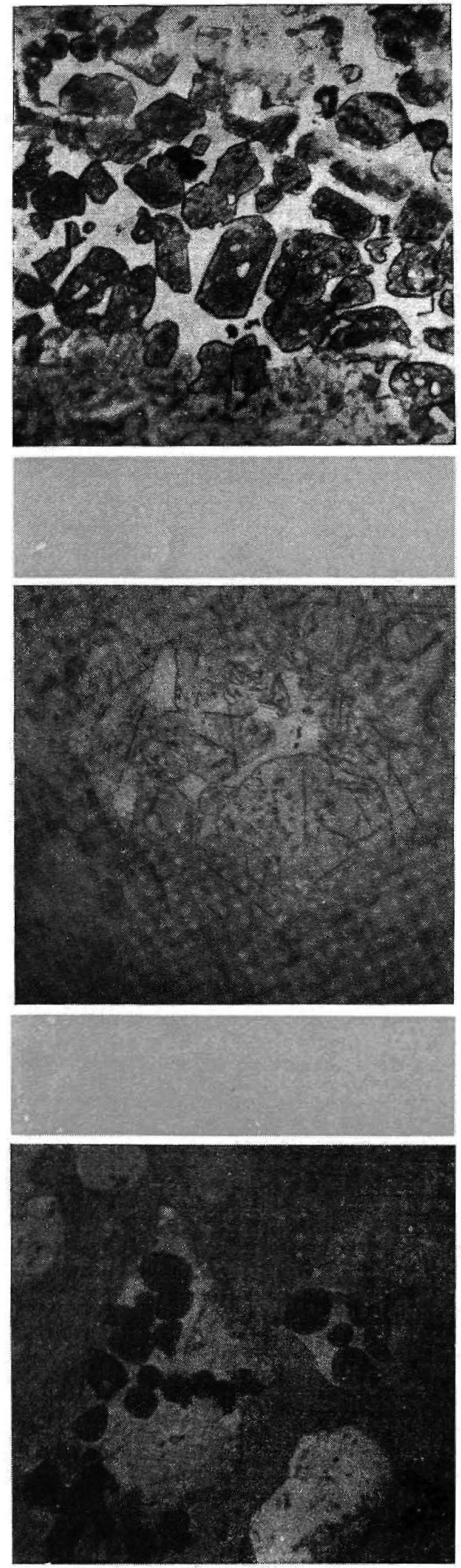

Fig. 17. GLINKER PARA CEMENTO PORTLAND NORMAL. Fase intersticial anormalmente abundante $y$ cristales irregularmente lormados, presentando una incipiente coalescencia. En el ángulo inferior izquierdo se nota un grupo de gránulo
de cal libre primaria.

Fig. 18. GLINKER PARA CEMENTO BLANCO.-Superficie rayada, común en los ataques por ácido bórico. Granos de avanzada coalescencia, dejando entre si campos de aluminate.

Fig. 19. Clinker Para CEMENTo BLANCO.-Aspecto de la cal libre por efecto del ataque por la solución de bórax. 


\section{Mieroseopios}

Trabajamos en el L. N. E. C. con dos microscopios metalográticos, ya que, como dijimos al principio, es el método de observación metalográfica que se aplica al clínker. El microscopio modelo Metatest, de la casa Reichert, con lámpara Lux TB para lluminación por reflexión, proporcionando una ampliación máxima de $512 x$, disponiendo de un juego de oculares $y$ objetivos, es un modelo de gran utilidad para una rápida observación de laboratorio, apropiado para el control de la marcha de una fábrica de cementos. Este modelo no permite obtener fotografias.

El microscopio universal "ME $\mathbf{F}$ ", también de Reichert, ya posee cámara fotográflca, con un poder de ampliación que llega a $2.000 x$. Posee filtros azul, verde, amarillo y negro, pudiendo funcionar por reflexión o por transparencla. Tiene posibilidad de adaptación de varios aparatos complementarios como son un polarizador y un microdurómetro, entre otros.

\section{Alblograffa}

(1) LEs, F. M.: Química del cemento y del hormigón. Edición revisada. Eđward Arnold, Edit, Madrid, 1960.

(2) Santaknld, L.; Elementos de quimica del cemento. Tamburini, Milano, 1960.

(3) Cononas, J. M,: Ténicas aplicadas a la investigaciơn del cemento. I, $\mathbf{F}_{*}$ T. c. c., Madrid, octubre 1950.

(4) Fưstkr Casas, J. M.: Técnicas y procedimientos petrograficos. I. E. T. c. c., Madrid, octubre 1950.

(5) Tavasci, B.: Observación microscópica del clinker con lua reflejada. I. E. T. c. c., Madrid, octubre 1950 .

(6) Paditla, E.: Alteraciones en la estruetura mineralógica del clinker sometido a un recocido prolongado. Industria Italiana del Cemento, octubre 1859.

(7) Tavasci, B., y Cereseto, A.: Sobre Ia determinación cuantitativa y cuallativa de la cal libre en el cemento portand. La Chimica e l'Industria, septiembre 1942.

(8) Aregre, R., y Tern Ima, P.: La microscopia del clinker. Aevue des Matériaux da Construction, junio 1957.

(9) Pratas, M.: Estudio microscópico de clinkeres y cementos nacionales. L. N. E. C., Lisboa, marzo 1955.

(10) Helt.ke, L., y TAYuor, M. F. W.: Datos ctistalográficos de los silicatos de calcio. Department of Scientifle and Industrial Research, Londres, 1956.

11) Trofer, F,: Conclusiones sobre el examen microscopico de diversos tipos de clinker de cemento portland. Zement-Kalk-Gips, septlembre $\mathbf{1 9 5 3 .}$

(12) SMith, D. K., MaJumdar, A. J., y OzDWaY, F.: Retisión del polfmorfismo del silicato bicalcico. Journal of the American Ceramic Society, Vol. 44, num. 8, agosto 1961. 